\title{
Chemical-Garden Formation, Morphology, and Composition. I. Effect of the Nature of the Cations
}

\author{
Julyan H. E. Cartwright, ${ }^{*}$ Bruno Escribano, ${ }^{* \dagger}$ and C. Ignacio Sainz-Díaz* \\ Instituto Andaluz de Ciencias de la Tierra, CSIC-Universidad de Granada, Facultad de Ciencias, E-18071 Granada, Spain
}

Supporting Information

\begin{abstract}
We have grown chemical gardens in different sodium silicate solutions from several metal-ion salts - calcium chloride, manganese chloride, cobalt chloride, and nickel sulfate-with cations from period 4 of the periodic table. We have studied their formation process using photography, examined the morphologies produced using scanning electron microscopy (SEM), and analyzed chemical compositions using $\mathrm{X}$-ray powder diffraction (XRD) and energy dispersive X-ray analysis (EDX) to understand better the physical and chemical processes involved in the chemical-garden reaction. We have identified different growth regimes in these salts that are dependent on the concentration of silicate solution and the nature of the cations involved.
\end{abstract}

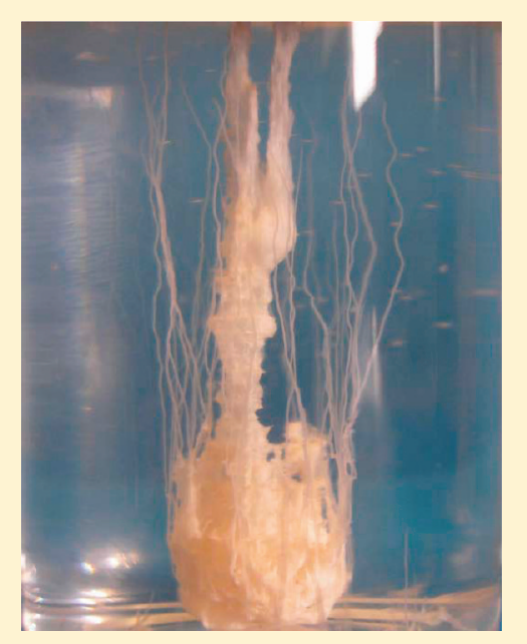

\section{INTRODUCTION}

Chemical gardens are the plantlike structures produced by a variety of different metal-ion salts when immersed in a solution of silicate or some other anions. ${ }^{1}$ When the salt crystal begins to dissolve in the silicate, it forms a gel around itself. This gel acts as a semipermeable membrane, allowing water from the silicate solution to flow in toward the salt crystal driven by osmotic pressure. As the water flows in and the crystal continues to dissolve, the pressure inside the membrane rises. At some point, the membrane ruptures, forming a hole through which the metal-ion solution from the inside flows out. This metal-ion solution reacts with the silicate outside, forming a tube around the flow by precipitation. Hence, its morphology is a product of forced convection driven by osmotic pressure through the semipermeable membrane and free convection due to buoyancy, since the ejected solution is generally lighter than the external silicate. The final result is generally a combination of tubes of different sizes and shapes, resembling a tree or a garden.

The main interest in them recently has been for educational purposes, but chemical gardens also have some fairly unexplored applications in processes of industrial importance that involve precipitation across a colloidal gel membrane separating two different aqueous solutions, for example, in the hydration of Portland cement ${ }^{2}$ and the corrosion of metals. ${ }^{3}$ It has been speculated that the membranes of chemical gardens produced in submarine vents may be an ideal site for the origin of life. ${ }^{4,5}$ There is not a great deal of published work that includes a thorough study of their morphology, chemical composition, and microstructure. $^{6-8}$ Different regimes of tube growth have been identified upon injecting a copper sulfate solution into sodium silicate. ${ }^{9-11}$ At lower concentrations of copper sulfate, there are found thin tubes that grow steadily: a regime termed jetting. At higher concentrations, growing tubes show pulsating behavior, called budding, in which the tip of the tube develops a bubble that inflates and bursts, nucleating a new droplet at the tube's end. The resulting morphology is one of wider globular tubes. We will use this jetting and budding terminology throughout this work.

The purpose of this article is to study the formation, morphology, and composition of tubes grown with several metal-ion salts, $\mathrm{CaCl}_{2}$, $\mathrm{MnCl}_{2}, \mathrm{CoCl}_{2}$, and $\mathrm{NiSO}_{4}$, with cations from period 4 of the periodic table, using different sodium silicate concentrations. The morphologies were studied using SEM imaging, and the compositional analysis was performed with EDX and $\mathrm{XRD}$, and some interesting differences between the cations are noted. A better understanding of the physics and chemistry of the processes of formation of chemical gardens should allow us to improve the control of the relative solution densities, osmosis, and diffusion, which is a step toward being able to fabricate microtubes with a desired morphology.

This work complements both a systematic investigation that we reported recently of the behavior of cations of group 2 in the

Received: October 18, 2010

Revised: January 9, 2011 
periodic table ${ }^{12}$ and experiments in microgravity on the same period 4 salts used in the present study that we report in part II of this paper. ${ }^{13}$

\section{EXPERIMENTAL METHOD}

Crystals of $\mathrm{CaCl}_{2}, \mathrm{MnCl}_{2} \cdot 4 \mathrm{H}_{2} \mathrm{O}, \mathrm{CoCl}_{2} \cdot 6 \mathrm{H}_{2} \mathrm{O}$, and $\mathrm{NiSO}_{4} \cdot 7 \mathrm{H}_{2} \mathrm{O}$ at analytical purity and homogenized with an agate mortar were used for our experiments. They were pressed into $200 \mathrm{mg}$ wafers of $1 \mathrm{~cm}$ diameter using a cell at 10 bar pressure during $10 \mathrm{~min}$. The use of the wafer permits more systematic and reproducible experiments by avoiding the problems of having different shapes for the initial conditions and, in the case of $\mathrm{CaCl}_{2}$, helps to inhibit salt hydration during handling at ambient humidity. Nevertheless, irregular grains of these salts were also used in some cases that we detail in the relevant section. The sodium silicate solutions were prepared from a commercial concentrated solution composed of $27 \% \mathrm{SiO}_{2}$ and $15 \% \mathrm{NaOH}$. They were diluted using bidistilled water to several concentrations between 6 and $0.3 \mathrm{M}$. The growth process at room temperature was followed for at least $24 \mathrm{~h}$, or for as long as was necessary for the salt to dissolve completely. The experiments were recorded using standard photographic equipment, after which the tubes were removed from the solution and were dried in air at $25{ }^{\circ} \mathrm{C}$. Most of the samples were not rinsed with water before drying, in order to avoid the dissolution of some components. However, some samples, as explained below, were rinsed in order to compare the possible changes arising from the rinsing operation.

Micrographs of the samples were obtained using a FEI Quanta 400 environmental scanning electron microscope (ESEM) operating at high vacuum and room temperature and a Leo Gemini 1530 field emission scanning electron microscope (FE-SEM). Chemical analysis of the solids was performed in situ in the microscope using EDX. Powder X-ray diffraction (XRD) analyses were performed in a PANalytical X'Pert PRO diffractometer with a wavelength of $1.5418 \AA$, and singlecrystal X-ray diffraction analyses were performed in a Siemens P4 diffractometer with a wavelength of $0.7107 \AA$. The tubes were separated from the rest of the chemical garden before performing X-ray powder diffraction, in order to avoid confusion with the amorphous pattern produced by the osmotic membrane. The identification of crystallographic phases in the diffractograms was performed with the Xpowder code, ${ }^{14}$ and the treatment of data from single-crystal XRD was performed with the XRD2DScan code. ${ }^{15}$

\section{RESULTS AND DISCUSSION}

We studied the growth process for all four salts at different silicate concentrations. In this series of experiments, we explore cations with different electronic properties. All these cations, one of group 2 and several transition element cations with different electron populations in the valence shell following the sequence $\mathrm{Ca}<\mathrm{Mn}<\mathrm{Co}<\mathrm{Ni}$, belong to the same period, period 4 , of the periodic table.

In general, the growth and formation of chemical gardens from the pressed wafers is drastically slower than with irregular grains of these salts, although the behavior is similar in both cases. We found a similar tendency but different behavior for the four salts with respect to the change of silicate concentration. At high concentrations, higher than 2 to $3 \mathrm{M}$, just one or two tubes grow. Depending on the seeding salts, these tubes may grow steadily or in a pulsating manner to a length of several centimeters. At lower concentrations, around $1 \mathrm{M}$, the salts grow a collection of multiple narrower tubes. We also found slower growth at these low silicate concentrations; the tubes showed a longer induction time to start growing and did not grow for too long.

Calcium Chloride. Gardens grown from calcium chloride show two distinct growth regimes at high and low silicate
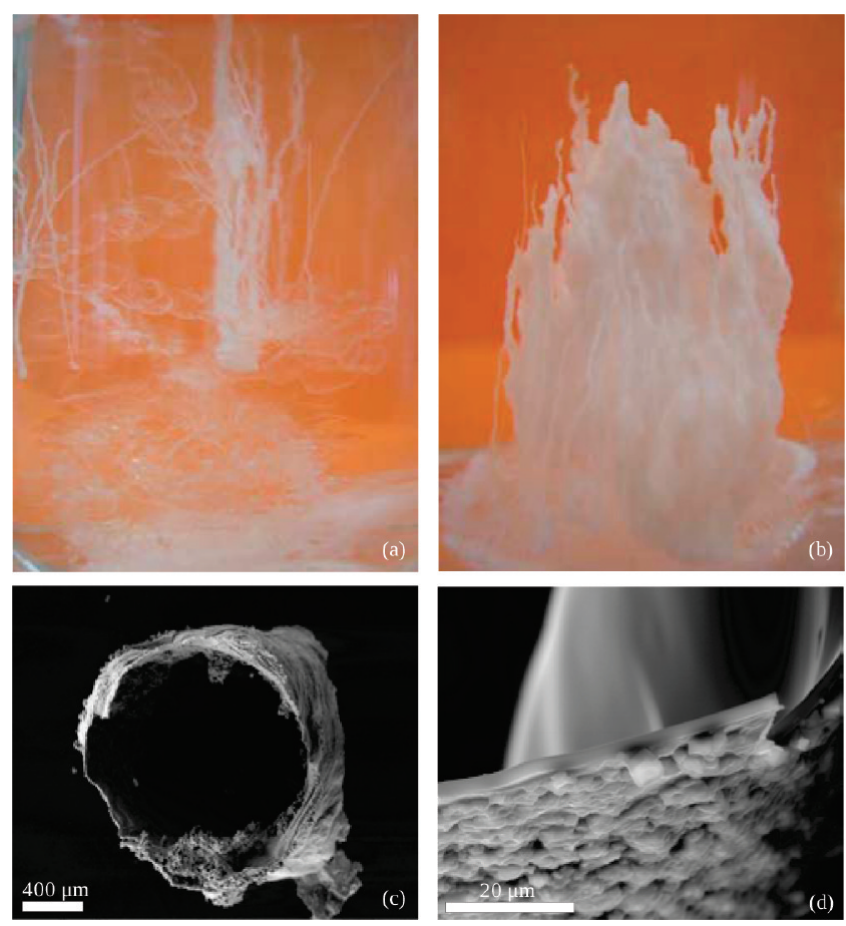

Figure 1. Growth of calcium chloride gardens with (a) $3 \mathrm{M}$ and (b) $1 \mathrm{M}$ silicate. The field of view is approximately $3 \mathrm{~cm}$ wide. (c) SEM image of a tube grown in $3 \mathrm{M}$ sodium silicate, with a wide section and thin walls. (d) External (smooth) and internal (rough) surfaces of the tube wall: $\mathrm{NaCl}$ crystals are observed on the internal surface.

concentrations. The general behavior starts with the formation of a gel membrane around the wafer, which then becomes a transparent "osmotic envelope" 12,16 formed by the entrance of external water through the membrane by osmosis.

At high concentrations of silicate ( 6 and $3 \mathrm{M})$, the membrane inflates by taking in water from the silicate solution under osmotic pressure until it becomes a transparent spherical elastic envelope that can reach $1-2 \mathrm{~cm}$ in diameter. Upon its rupture, only one or two wide tubes grow out of the membrane. The envelope's walls can be as thick as $1 \mu \mathrm{m}$, and it may take from 1 to $5 \mathrm{~min}$ to burst and for the first tube to grow. As the envelope inflates, it may trigger the growth of transparent fingers, wide transparent twisted tubes, or narrow white tubes. Usually, the wider tubes, with a diameter of 1 to $3 \mathrm{~mm}$, form first. They grow in periodic pulses, often changing direction with every pulse, with a period that can range from under one second up to one minute, following what Thouvenel-Romans et al. designate the budding regime ${ }^{9}$ induced by pressure oscillations in the osmotic envelope. This regime only allows one or two tubes to grow, these tubes being the widest of all salts studied here and growing rather rapidly, around $3 \mathrm{~mm}$ per minute. During the handling procedure, we appreciated that these tubes are highly flexible. When the growth of these transparent tubes ceases, narrow white tubes start growing from them (Figure 1a). In general, all transparent forms turn white after one day or more; we surmise that layers of silicate are deposited slowly during aging. It appears that the strength and elasticity of the membrane impede the rupture of the envelope with $6 \mathrm{M}$ silicate, and often only some random deformations or finger formations are observed. According to our previous calculations, ${ }^{12}$ the maximal density of the inner solution is lower than that of the external solution. In some experiments, when the salt is completely dissolved and the pseudospherical 
envelope is accidentally detached from the bottom of the reactor, buoyancy pushes up the whole envelope to the top without it breaking. In other experiments, we noted that the high viscosity of the $6 \mathrm{M}$ silicate can facilitate the presence of air bubbles trapped close to the surface of the initial salt seed during the preparation of the experiment. An air bubble enhances the formation of a wide transparent tube. ${ }^{10,17,18}$

At lower silicate concentrations, around $1 \mathrm{M}$, the seeding salt fails to develop an observable osmotic envelope as it does at higher concentrations. Instead, multiple narrow tubes grow straight out from the initial salt crystal. These tubes are all narrower than $0.1 \mathrm{~mm}$ and grow together by the hundreds in a tightly bound opaque cluster. As the silicate is more dilute, the growth rate of these narrow tubes is much slower than those discussed above, about $1 \mathrm{~mm}$ per minute. The fact that these tubes appear white instead of transparent may be a consequence of their narrow diameter and thicker wall and the clustering of the tubes (Figure 1b); there is no compositional difference with respect to those from more concentrated silicate, as we shall see below.

With high-concentration silicate, the envelope swells with water, increasing its volume; this does not occur at lower concentrations. This is despite the fact that the difference in osmotic pressure across the membrane will be lower for higher silicate concentrations. This difference in behavior could be due to the mechanical properties of the silicate membrane formed around the salt crystal: more concentrated silicate solutions apparently produce more resistant membranes, and so, they may sequester more water inside and at higher pressures before rupturing. At lower silicate concentrations, the osmotic membrane will possibly be too weak to support such internal pressure. Hence, the envelope will be smaller, and this membrane will break in many places. In this case, the inner solution is expelled in a jetting growth regime, precipitating the silicate and forming the white tubes. The wide transparent tubes observed at high silicate concentration are not produced in the same way. When the osmotic and buoyancy forces are strong enough, the osmotic membrane stretches and breaks, concurrent with the formation or budding of a new extension of the membrane with the flow of the inner solution. This extension of the membrane increases the volume and the internal pressure is released. This produces a cessation of the flow, closing the hole in the membrane. Again, the osmotic flow increases the inner pressure and the freshly formed membrane will break, following a random direction, and forming another segment of the tube wall. The budding growth tends to be periodic, facilitating a slow and regular formation of the tube walls and yielding bulging but unbranched tubes. These tubes may be more or less vertical; the presence of an air bubble enhances the buoyancy forces and the tubes produced with an air bubble will be more vertical and uniform.

We used electron microscopy to analyze the morphological differences between tubes formed in the two regimes of jetting and budding. In the case of $3 \mathrm{M}$ silicate, we measured diameters from $500 \mu \mathrm{m}$ to $1 \mathrm{~mm}$, with wall thicknesses from 1 to $10 \mu \mathrm{m}$ (Figure 1c) for the transparent budding tubes. The appearance of the surfaces under SEM shows that the textures are clearly different for the internal and the external sides of the tubes, being smooth on the outside and rough on the inside (Figure 1d). EDX analysis on both sides shows a much higher proportion of oxygen on the inside, indicating the presence of calcium oxides-hydroxides along with $\mathrm{CaSiO}_{3}$. Analysis of the external side shows more silicon, indicating that the external wall surface is composed mainly of $\mathrm{CaSiO}_{3}$. We also found small $(\sim 1$ $\mu \mathrm{m}$ ) crystals of $\mathrm{NaCl}$, especially on the inside surface of the tube walls, which implies that $\mathrm{Na}^{+}$ions can also cross the osmotic membrane. The presence of $\mathrm{NaCl}$ has not been reported by other authors in these systems, probably because they rinsed the samples. We detected the presence of small crystals of $\mathrm{NaSiO}_{3}$ on the external surface arising from the evaporation of the external solution (Figure 1a in Supporting Information (SI)) and the presence of $\mathrm{SiO}_{2}$ attached to the external surface of the tubes after aging (Figure $1 \mathrm{~b}$ in SI). X-ray diffraction analysis of calcium tubes did not show any significant crystallinity except for $\mathrm{NaCl}$ reflections, indicating, according to our previous work, ${ }^{12}$ that the nanostructure is mainly disordered.

Manganese Chloride. With manganese chloride, the variation of behavior with respect to the silicate concentration follows a similar tendency to the calcium case; however, some morphological differences were observed. With $6 \mathrm{M}$ silicate, as in the calcium case, the osmotic envelope shows little tendency to form tubes; however, the volume of the osmotic envelope is smaller than in the calcium case. As with calcium, the presence of an air bubble promotes the formation of a wide straight tube of some $3 \mathrm{~mm}$ diameter. The buoyancy forces push up the air bubble, and the surface tension of the water in the contact with the air bubble on the rim of the tube enhances a continuous and uniform contact between the internal and external solutions producing the straight and homogeneous tube morphology. ${ }^{10,17,18}$ Hence, a wide translucent straight tube is formed following the trajectory of the air bubble through the high-viscosity silicate solution (Figure 2a in SI). With $3 \mathrm{M}$ silicate, only one or two wide tubes are formed following a budding regime. ${ }^{9}$ In this case, the osmotic envelope is also very small, the wide tubes are translucent with an ivory color, instead of transparent as with calcium, and they are less wide than in the calcium case. When the growth of these budding tubes is interrupted (Figure 2a,b), the osmotic envelope continues growing, and later, new narrow, opaque tubes begin growing from the previous ones. Some fingers were also observed growing directly from the osmotic envelope (Figure 2c,d).

With $2 \mathrm{M}$ silicate, wide budding tubes also grow at a faster growth rate than in the above case (Figure 2e). When the seed is immersed in $1 \mathrm{M}$ silicate, the tube growth rate is fastest for the manganese series and narrow opaque tubes grow grouped in clusters (Figure $2 \mathrm{f}$ ). They grow more vertically than the above budding tubes and with a faster linear rate, approximately 5$6 \mathrm{~mm}$ per minute. The osmotic envelope adopts the shape of the wafer, and the wafer seems to swell slightly and is empty inside at the end of the reaction. A group of tubes at the edge of a wafer grows more rapidly than in the other zones of the wafer, and any irregularity at the edges of the wafer due to the preparation procedure enhances the growth (Figure 2b, c in SI). This is presumably due to the curvature of the osmotic envelope at the wafer's edge. This enhancement of growth is also observed when the wafer comes in contact with the reactor walls and a group of tubes grows attached to the reactor wall. With $0.6 \mathrm{M}$ silicate, the morphology is similar to that at $1 \mathrm{M}$ but with a longer induction time and a lower growth rate (Figure $2 \mathrm{~d}$ in SI).

Throughout the manganese series, the initial pale rose color of the wafer turns to an ivory-white color in the osmotic envelope and in the tubes, and after one day, these solids turn brown. At the end of the growth process, the interior of the wafer is dissolved, the wafer becomes empty, and only the external surface is maintained. In spite of the heterogeneity in the shape and size of tubes, we can estimate some averaged values of tube 

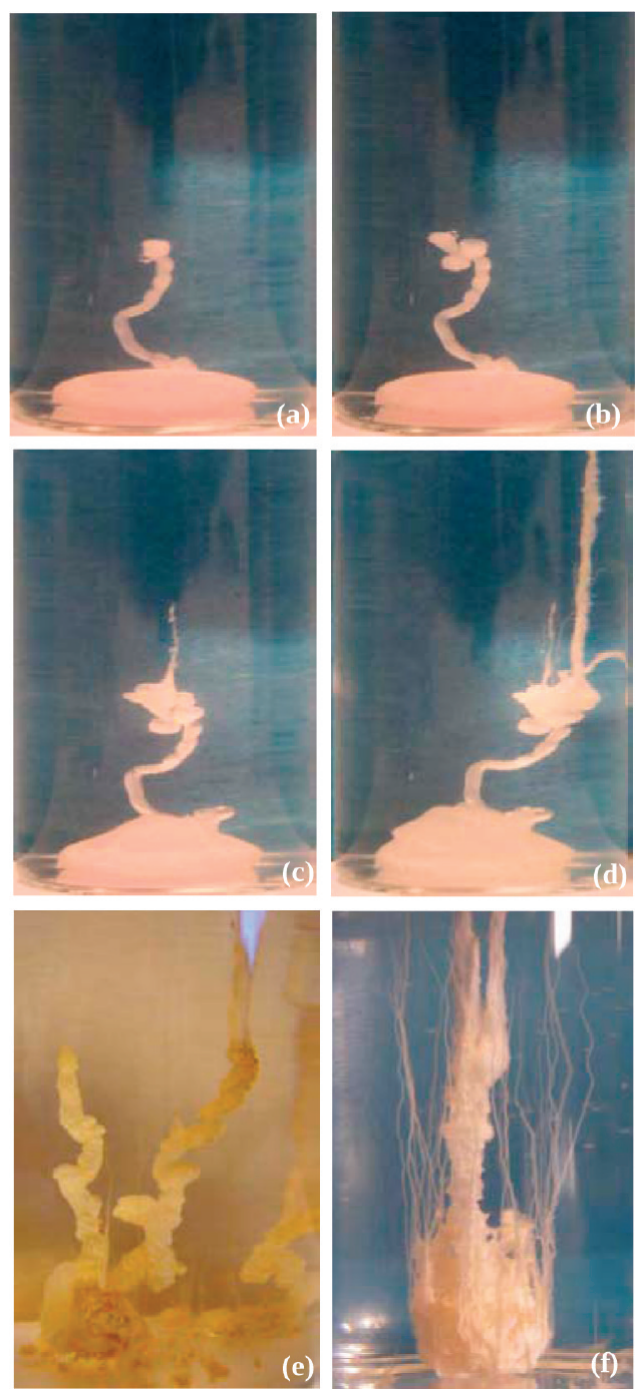

Figure 2. Manganese chloride garden growth in $3 \mathrm{M}$ silicate with snapshots of a growth sequence shown at (a) $5 \mathrm{~min}$, (b) $10 \mathrm{~min}$, (c) 30 $\mathrm{min}$, and (d) $50 \mathrm{~min}$. Other examples with (e) $2 \mathrm{M}$ and (f) $1 \mathrm{M}$ silicate concentrations. The field of view is approximately $3 \mathrm{~cm}$ wide.

diameter, and we find that there is a direct relationship between the silicate concentration and the diameter (Figure 3).

For both salts, calcium and manganese, the budding regime of tube growth is observed with high concentrations of silicate solution $(>2 \mathrm{M})$, while the narrow opaque tubes grow with more dilute silicate. However, the behavior is different for each salt. The lowest induction time, highest number of tubes, and highest linear growth rate in the formation of tubes is with $3 \mathrm{M}$ silicate for calcium, whereas it is with $1 \mathrm{M}$ silicate for manganese. The osmotic envelope with concentrated silicate $(>2 \mathrm{M})$ is much larger for calcium than for manganese, with more water entering the inner solution. On the contrary, the mechanical stability of the gardens with dilute silicate $(<1 \mathrm{M})$ is higher for manganese than for calcium.

In Figure 4a, we show the micromorphology of the budding tubes grown in $3 \mathrm{M}$ silicate. As in the calcium case, the outer surface texture of the budding tubes is smooth, whereas the inner surface is rough. EDX analysis indicates the presence of manganese silicate on the outer side and manganese oxide-hydroxide on the inner. In the inner surface of the tubes close to the bottom,

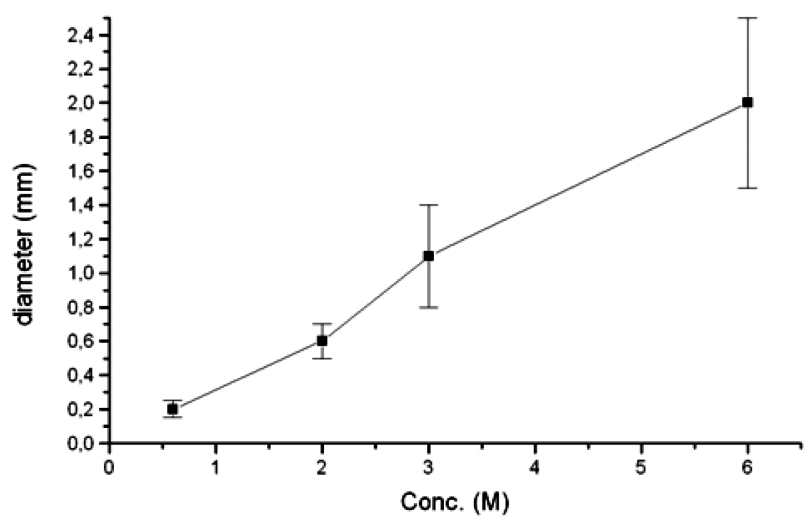

Figure 3. Relationship between the silicate concentration and the average value of the tube diameter for manganese chloride.

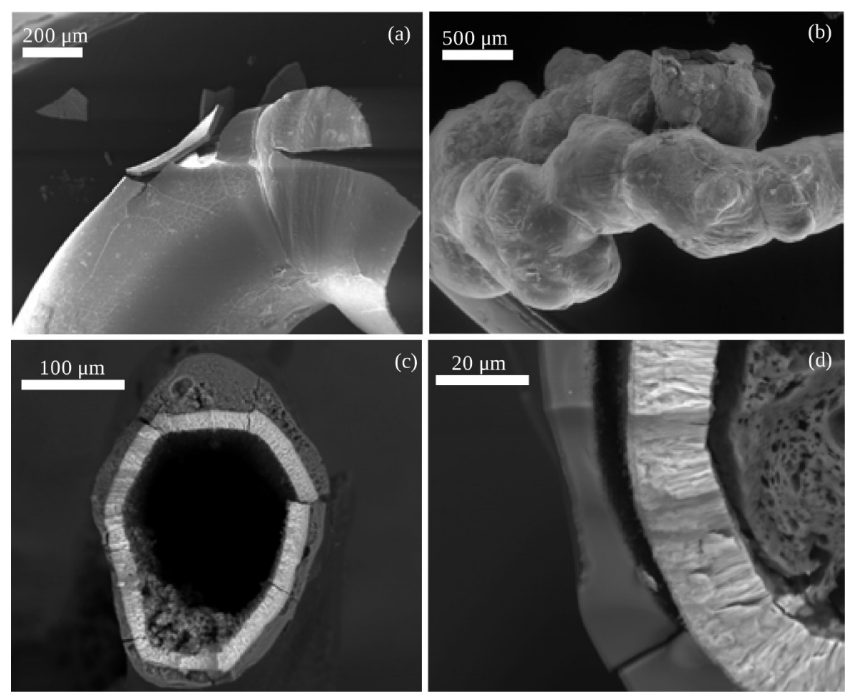

Figure 4. SEM images: Budding tube grown from manganese chloride in (a) $3 \mathrm{M}$ and (b) $2 \mathrm{M}$ sodium silicate. (c,d) Image of straight, narrow tube grown from manganese chloride in $0.6 \mathrm{M}$ silicate taken using a backscattering solid-state detector.

near the osmotic envelope, a significant amount of manganese chloride is detected. Typical tube diameter is $\sim 1 \mathrm{~mm}$, with a wall thickness of $\sim 10 \mu \mathrm{m}$. The micromorphology and chemical composition is similar for the budding tubes grown in $2 \mathrm{M}$ silicate. In Figure 4b, we can observe the irregular morphology of a budding tube, which presents a rough inner surface and a smooth outer surface (Figure 3a in SI). The texture of the narrow opaque white tubes grown in $0.6 \mathrm{M}$ is different: The walls of the isolated opaque narrow tubes are thicker $(40-60 \mu \mathrm{m})$ than in the budding ones (Figure 4c,d). In these opaque narrow tubes, the interfaces between layers with different chemical composition are sharper than in the tubes from a budding regime. By means of a backscattering detector, we can distinguish the brighter zones of manganese oxide-hydroxide and the external dark zone of silicate (sodium and manganese) and silica (Figure 4c). These layers are not chemically joined to one other and they separate from each other after drying (Figure 4d). The wall thickness is greater in the tube clusters than in the isolated tubes (Figure $3 \mathrm{~b}$ in SI). EDX chemical analysis indicates that the interstitial zone between tubes in the clusters is formed mainly of sodium silicate. 
a

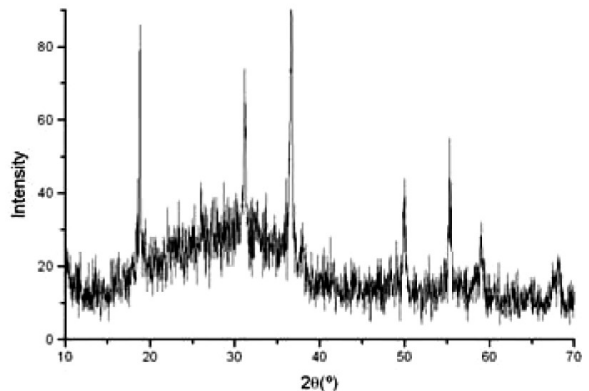

$\mathrm{b}$

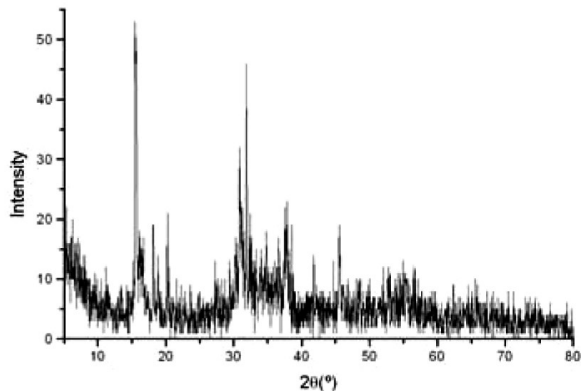

C

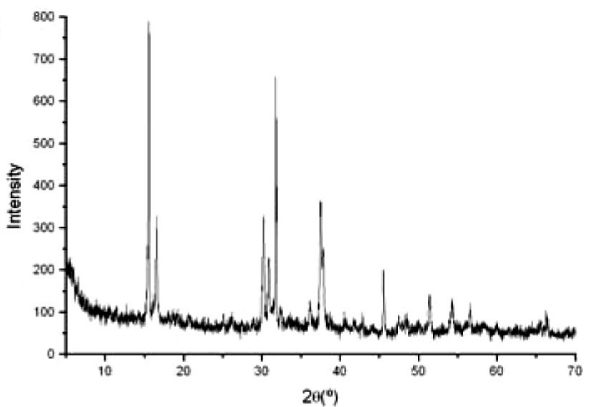

Figure 5. X-ray powder diffraction patterns of tubes grown from manganese chloride for (a) budding tubes; (b) cluster of straight narrow tubes in $2 \mathrm{M}$ silicate; (c) tubes grown from wafers in $3 \mathrm{M}$ silicate.

The manganese series produces higher crystallinity growths than we found with calcium. We identified manganese hydroxide crystals in most experiments (Figure 5). In the budding tubes, the main crystalline component is $\mathrm{MnO} \cdot \mathrm{H}_{2} \mathrm{O}$ with peaks at $18.7^{\circ}$

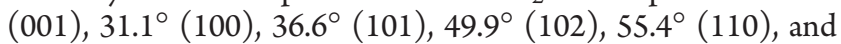
$58.9^{\circ}(111)$ ( $2 \theta$ units) (Figure $\left.5 a\right)$, whereas in the opaque clusters of narrow tubes, we detected crystals of $\mathrm{NaCl}$ (reflections at $31.8^{\circ}(200), 45.6^{\circ}(220), 56.4^{\circ}(222)$, and $66.3^{\circ}(400)$, hydrated $\mathrm{MnCl}_{2}$ (reflections at $15.5^{\circ}(011), 16.5^{\circ}$ (110), $30.1^{\circ}$

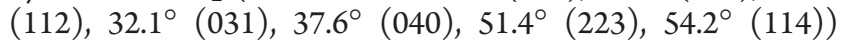
(Figure $5 b, c)$ and a small proportion of $\mathrm{MnO} \cdot \mathrm{H}_{2} \mathrm{O}$ (Figure $5 \mathrm{~b}$ ).

In order to explore the crystallinity and ordering of the crystals in the tubes, we analyzed an opaque cluster of tubes with a length of $3 \mathrm{~cm}$ directly by single-crystal XRD. Two zones were analyzed: the center and the border of the cluster. The two-dimensional patterns of both zones show a uniform distribution of the reflections indicating the small size and random orientation of the crystals. In the border of the cluster, some large bright spots are observed indicating the existence of larger crystals in this zone. Probably, this is due to their having a lower growth rate. The analyses of both zones show similar diffractograms and crystalline compositions, being mainly manganese oxide-hydroxide and $\mathrm{MnCl}(\mathrm{OH})_{n}$ (Figure 6).

Cobalt Chloride. Cobalt chloride shows a similar behavioral dependence with silicate concentration to that of the previous
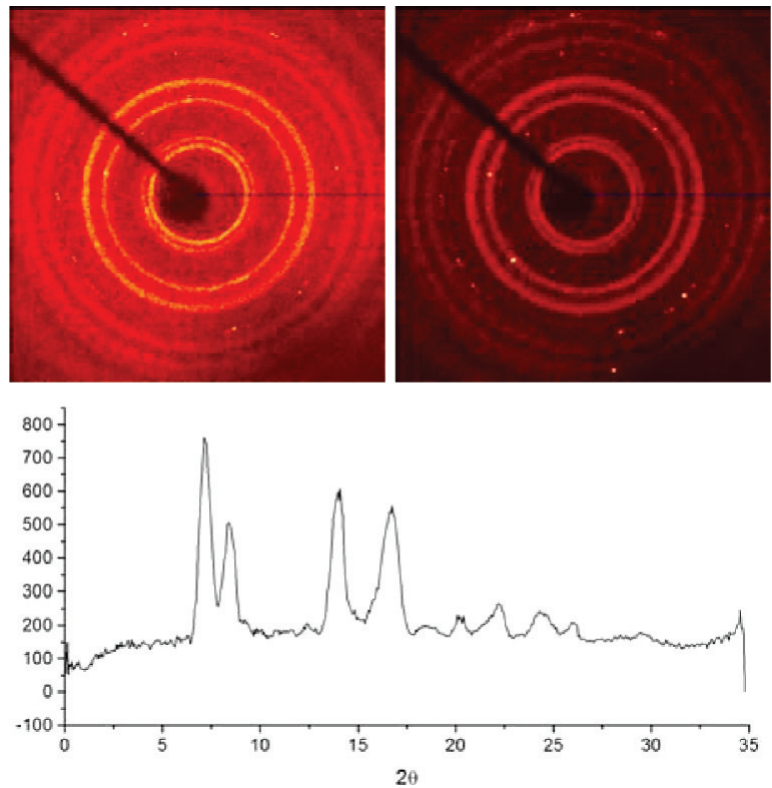

Figure 6. X-ray diffraction of one tube grown from manganese chloride in $2 \mathrm{M}$ silicate. Two-dimensional XRD pattern in (a) the center and (b) the border of the tube. (c) $2 \theta$ scan integrated from the $2 \mathrm{D}$ pattern of the center of the tube. $(\lambda(\mathrm{Mo})=0.7107 \AA)$.

salts: wide tubes at high silicate concentration and narrow tubes at lower silicate concentration. In general, the cobalt salt has a much lower induction time and higher linear growth rate than the calcium and manganese ones.

In Figure 7, we follow in detail the growth sequence of one particular cobalt chloride tube in $3 \mathrm{M}$ silicate. We cannot observe the formation of a clear osmotic envelope around the wafer at the beginning, although at the end, the wafer is hollow and the envelope adopts the shape of the wafer. The presence of an air bubble facilitates the generation of a straight wide tube that grows upward, since buoyancy forces are important and are formed following a budding regime, ${ }^{10,17,18}$ at a rate of $1 \mathrm{~cm}$ per minute. The wafer and the initial part of the tube are purple, but the top of the tube becomes green (Figure $7 \mathrm{~b}$ ). After a while, the air bubble disappears, growth stops, and later, the tube continues growing with a blue color (Figure 7c). The growth stops again, and a small envelope is formed on the top of the tube, from which clusters of narrow blue tubes grow together. As the cluster continues growing, its accumulated weight bends the original tube until it leans against the container wall. When this tube reaches the top surface, small green budding tubes grow along the top surface of the wafer and a plume of clusters of blue narrow tubes starts growing from these budding tubes and from the rim of the wafer.

In all wafers, the tubes start growing at the rim of the wafer. Starting from irregular grains, the induction time is lower, and there are a greater number of tubes and a higher linear growth rate than with the pressed wafers. At high concentrations of silicate ( 6 and $3 \mathrm{M}$ ), the growth of tubes with a budding regime is observed at the first stage of the reaction. At lower concentrations of silicate ( 1 and $0.6 \mathrm{M})$, clusters of narrow tubes are formed. With very dilute silicate solution $(0.3 \mathrm{M})$, the induction time is higher, there is a lower density of tubes and a slower linear growth rate, and individual narrow tubes are formed with a budding regime with pseudohelicoidal patterns (see Figure 8 and also Figure 4 in SI). In most cases, the osmotic envelope maintains the pink color of a $\mathrm{Co}^{2+}$ cation that is octahedrically coordinated. 


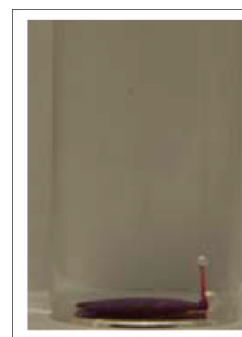

$3 \mathrm{~min}$.

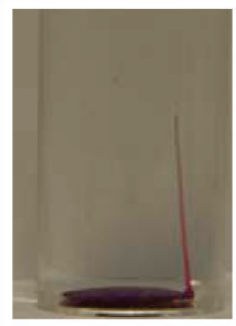

$4 \mathrm{~min}$

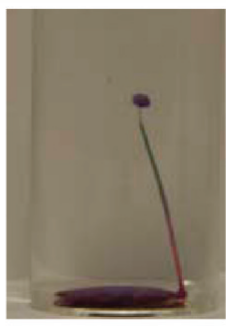

6 min.

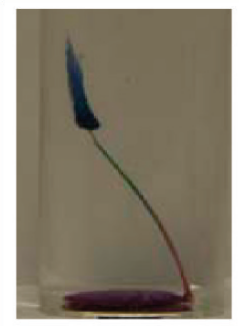

8 min.

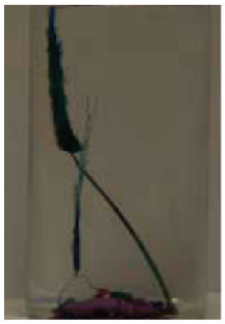

$10 \mathrm{~min}$.

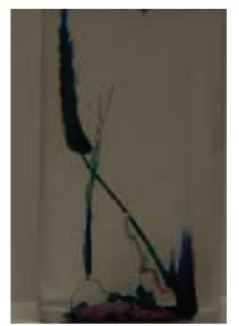

$25 \mathrm{~min}$.

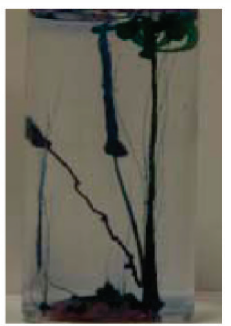

$40 \min$.

Figure 7. Cobalt chloride garden grown in $3 \mathrm{M}$ sodium silicate. The field of view is approximately $3 \mathrm{~cm}$ wide.
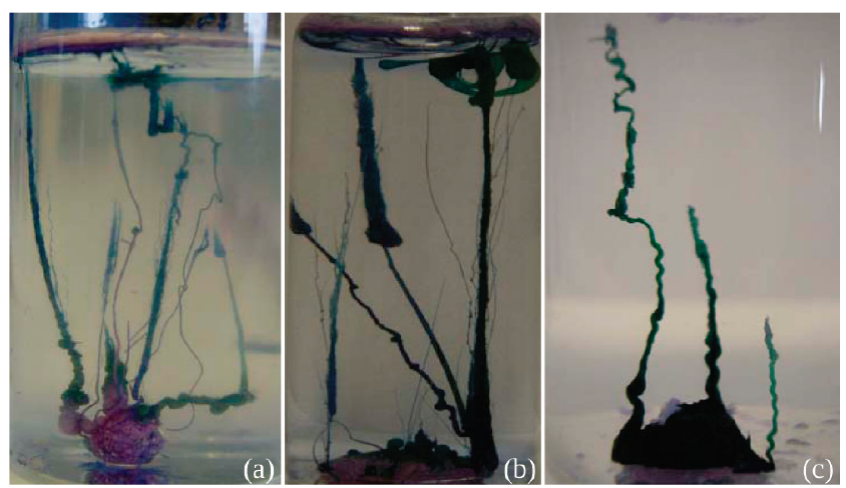

Figure 8. Comparison of different completed growths of cobalt chloride in (a) $6 \mathrm{M}$, (b) $3 \mathrm{M}$, and (c) $0.3 \mathrm{M}$ silicate. The field of view is approximately $3 \mathrm{~cm}$ wide.

In the tubes, the amount of cobalt oxide-hydroxide and the tetrahedral coordination increase showing a blue - green color.

In Figure 9a, we show an SEM image of the cluster of tubes discussed above. The cluster is composed of dozens of tubes with individual diameters under $50 \mu \mathrm{m}$. In Figure $9 \mathrm{~b}$, we observe tubes growing from spheres formed on top of the wafer surface at the first stage of growth, forming a cluster of many tubes growing upward (Figure 5 in SI). While we do not know the origin of the spheres, the growth of the tubes from them recalls the work of Makki et al., ${ }^{17}$ who described similar microtubes growing from beads. The tubes grow along the surface of the neighboring tubes; probably, the surface enhances the nucleation of the silicate forming the walls of the tube, as in the case of the previous clusters of tubes, favoring this kind of growth instead of the formation of isolated tubes. The texture is different in the outer and inner parts of the tubes, being smooth outside and rough in the inner surface (Figure 9c). The chemical composition is also different, being mainly cobalt silicate in the smooth outer layer and cobalt oxide-hydroxide in the rough inner layer. On this inner surface, many spheroidal rosettes of crystals formed of cobalt oxide-hydroxide are observed (Figure 9d). This morphology has been also detected by others in other cobalt oxides. $^{19}$

$\mathrm{X}$-ray diffraction showed that these tubes are mostly disordered in their microstructure. Only in some cases did we find cobalt hydroxide crystals (Figure 6 in SI) with reflections at $19.0^{\circ}$ (001), 32.4 ${ }^{\circ}(100), 37.9^{\circ}(101), 38.6^{\circ}, 51.3^{\circ}, 57.8^{\circ}(110), 61.4^{\circ}$, $69.4^{\circ}$, and $71.2^{\circ}$ ( $2 \theta$ units) (Figure $6 \mathrm{~b}$ in SI). In some tubes, crystals of $\mathrm{NaCl}$ can be detected with reflections at $27.5^{\circ}, 31.8^{\circ}$, $45.4^{\circ}$ (Figure $6 \mathrm{a}$ in SI). Infrared analysis of cobalt chloride tubes
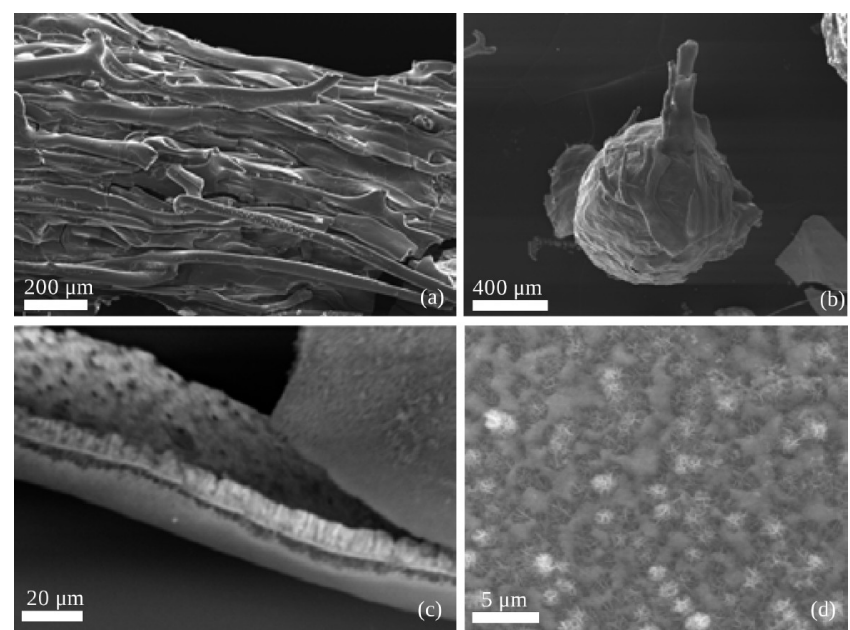

Figure 9. SEM micrographs of a (a) cluster of narrow tubes grown from cobalt chloride in $3 \mathrm{M}$ sodium silicate; (b) tubes growing from spheres on the wafer surface in $3 \mathrm{M}$ silicate in an initial stage; and a (c) wall of tubes grown from cobalt chloride in $1 \mathrm{M}$ silicate. (d) Detail of the inner surface of the tube wall.

grown in $1 \mathrm{M}$ silicate shows an intense narrow peak at $3551 \mathrm{~cm}^{-1}$ characteristic of $v(\mathrm{OH})$ of $\mathrm{OH}$ groups joined to cobalt ${ }^{20}$ indicating the presence of $\mathrm{Co}(\mathrm{OH})_{2}$ (Figure 7 in SI). This band was not observed in the other salts.

Nickel Sulfate. Nickel sulfate displays the longest induction time and lowest linear growth rate of all the salts studied, but produces the most robust tubes with the thickest walls. With highly concentrated silicate, narrow isolated straight tubes grow vertically upward. Some of these tubes show a capsule at the top, probably covering an air bubble that facilitates growth (Figure 10a). With $3 \mathrm{M}$ silicate, narrow isolated tubes grow forming a curve starting upward and later bending to a horizontal direction; probably, the buoyancy forces are not strong enough to sustain vertical growth (Figure 10b). With more dilute silicate $(<2 \mathrm{M})$, these isolated tubes are not formed, but instead an irregular cluster of tubes (Figure 10c). An osmotic envelope is not observed, and the increase in volume of the wafer is smaller than in the previous series; a growth sequence is displayed in Figure 11. Initially, a short and relatively wide budding tube grows with a light-green color that later continues growing as a possible jetting regime forming an isolated tube that, as in the above instance, does not grow vertically but curves. Later, some wide tubes grow along the surface of the wafer from the borders to the center from where a wide cluster of tubes grows straight upward. 
In highly concentrated silicate, small budding tubes are formed in the initial state, whose micromorphology shows that they are highly twisted (Figure 12a). The narrow isolated tubes grown with $3 \mathrm{M}$ silicate can be of $200 \mu \mathrm{m}$ diameter and show a smooth outer surface (Figure 12b). The micromorphology of the fibers observed in $1 \mathrm{M}$ silicate shows a highly disordered cluster of microtubes (Figure 8a and b in SI) where the thickness of the wall is about $100 \mu \mathrm{m}$. We can see that the thickness of its walls are the highest among all studied salts. This morphology is different from that seen in all other salts. EDX analysis on the walls shows higher silicon content toward the exterior and higher nickel and oxygen content toward the interior, indicating $\mathrm{NiSiO}_{3}$ outside and nickel oxide-hydroxide inside. Pictures taken with the backscattering detector show zones with different brightness in this tube section. We note a dark external layer of sodium silicate, a bright zone of nickel silicate, and an internal zone with medium brightness with a higher amount of nickel oxide (Figure $8 \mathrm{~b}$ in $\mathrm{SI}$ ).

Crystals of sodium sulfate in the form of flakes are observed on the inner surface of the osmotic envelope and the lower part of the tubes (Figure $8 \mathrm{c}$ in SI), indicating that sodium ions can cross the osmotic membrane. In Figure 12c, we can see the remains of the osmotic membrane. It is $50-100 \mu \mathrm{m}$ thick and is covered with a layer of silica on the outside. Its inner surface is scattered with hollow spheres of $20-120 \mu \mathrm{m}$ diameter (Figure $8 \mathrm{~d}$ in SI) and thin walls (approximately $1-5 \mu \mathrm{m}$ ) that can be broken (Figure 12d) during the drying process. These hollow nanospheres appear also on the inner surface of some tubes (Figure 12e) and are composed of nickel silicate with a high content of nickel that we can associate with a nickel hydrosilicate, such as $\mathrm{Ni}_{3} \mathrm{Si}_{2} \mathrm{O}_{5}(\mathrm{OH})_{4}$, according to other hollow nanospheres obtained with nickel. ${ }^{21}$ Such spheres were not detected with the other salts.

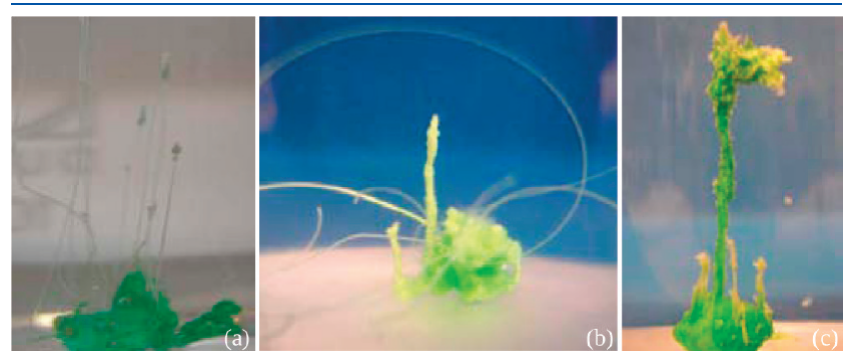

Figure 10. Nickel sulfate gardens growing in (a) $6 \mathrm{M}$, (b) $3 \mathrm{M}$, and (c) 1 $\mathrm{M}$ silicate. The field of view is approximately $3 \mathrm{~cm}$ wide.
The better-crystallized phases found in chemical gardens grown from nickel sulfate were sodium sulfates, which again show that sodium ions do cross the osmotic membrane and participate in the building of the tube walls (Figure 9 in SI)). Poorly crystallized hydrated nickel hydroxide can be identified according to previous XRD studies. ${ }^{21}$ We also identified some reflection peaks assigned to nickel hydrosilicate, $\mathrm{Ni}_{3} \mathrm{Si}_{2} \mathrm{O}_{5}(\mathrm{OH})_{4}{ }^{22}$ A similar composition was detected in nickel sulfate gardens grown with 3 and $1 \mathrm{M}$ silicate.

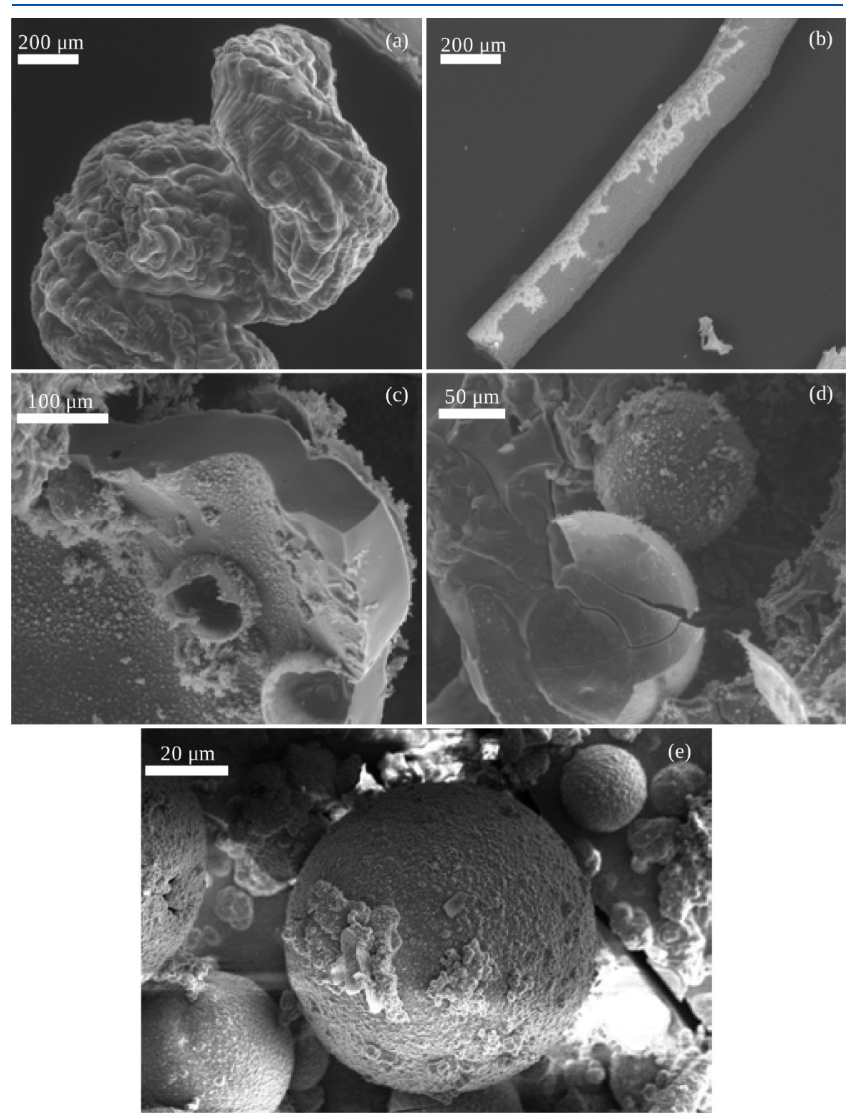

Figure 12. SEM micrographs of (a) nickel sulfate budding tube grown from grains in $6 \mathrm{M}$ silicate; (b) nickel sulfate tube grown from grains in 3 $M$ silicate; the white stains are remains of sodium silicate; (c) section of the osmotic envelope of a nickel sulfate tube grown from grains in $1 \mathrm{M}$ silicate; the internal surface is covered with spheres; (d) some of the spheres attached to the inner side of the membrane; the broken one shows their hollow nature; (e) microspheres formed on the inner surface of a tube.
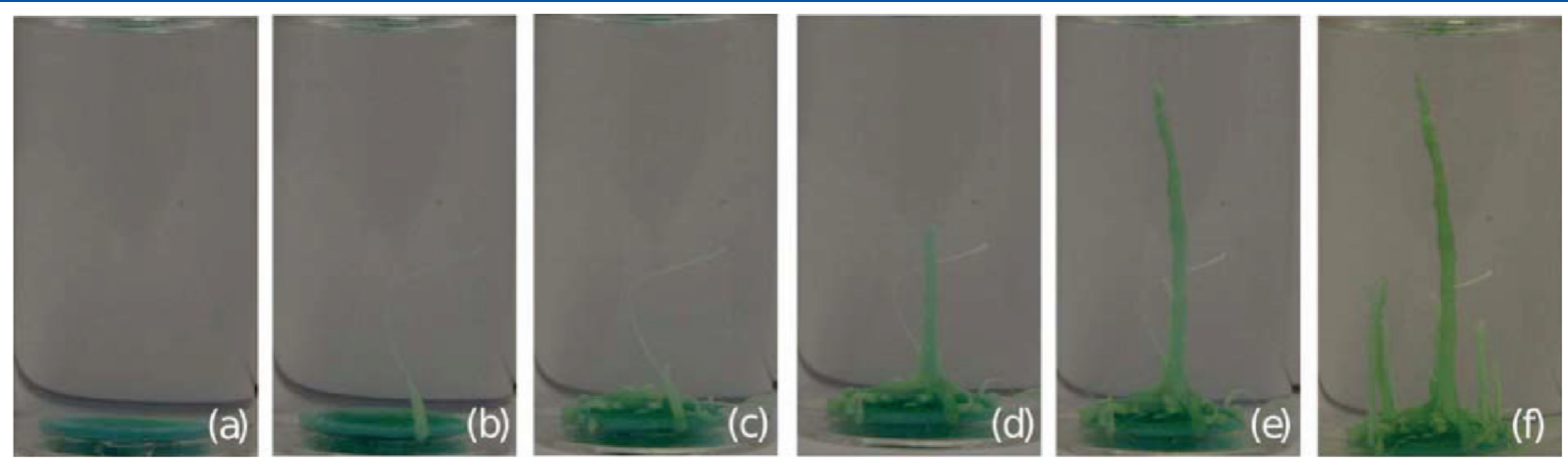

Figure 11. Snapshots of the growth of a chemical garden from a pressed wafer of nickel sulfate in $3 \mathrm{M}$ silicate after (a) $1 \mathrm{~min}$, (b) $15 \mathrm{~min}$, (c) $30 \mathrm{~min}$, (d) $35 \mathrm{~min}$, (e) $40 \mathrm{~min}$, and (f) $60 \mathrm{~min}$. The field of view is approximately $3 \mathrm{~cm}$ wide. 


\section{CONCLUSIONS}

We found distinct growth regimes in different sodium silicate solutions. Lower concentrations produce clusters of narrow tubes, while higher concentrations produce single and wider tubes. This is a general behavior for all the studied salts, but with some differences. Calcium chloride has the lowest induction time and highest linear growth rate, growing wider tubes with flexible thin walls. Nickel sulfate on the other hand has the slowest growth rate, producing very robust tubes with the thickest walls of all studied salts. Manganese and cobalt chloride have intermediate behavior. This tendency can be related with the solubility of the salts, e.g., in hydroxides the solubility product constant is $5 \times 10^{-6}, 2 \times 10^{-13}, 1.6 \times 10^{-15}$, and $5 \times 10^{-16}$ for calcium, manganese, cobalt, and nickel species, respectively. ${ }^{23}$

The swelling of the initial osmotic envelope decreases following the sequence $\mathrm{Ca}>\mathrm{Mn}>\mathrm{Co}>\mathrm{Ni}$. The swelling can be related with the solubility of the seed salts as detailed above; the more soluble the salt, the greater will be its osmotic pressure. The inflation of the membrane must also be related with its elasticity, strength, and permeability, which factors could be related with the $d(\mathrm{M}-\mathrm{O})$ bond length that follows the same sequence $\mathrm{Ca}>$ $\mathrm{Mn}>\mathrm{Co}>\mathrm{Ni}$. This ordering is inversely related with the electron population in the valence shell of the cations.

The various specific colors of the chemical gardens for the different salts show that they are not formed merely of sodium silicate precipitating under the effect of the low $\mathrm{pH}$ of the internal solution, but by the combination and coprecipitation of the silicate anion with the cation of the seeding salt, with the forming cation providing the color. Our chemical analyses confirm this conclusion.

Electron microscope imaging showed that tube walls are not homogeneous across their section. There is both a morphological and a compositional difference between the inner and outer surfaces. The different morphologies indicate that the tubes are formed by two layers, metal oxide-hydroxide on the inside and metal silicate on the outside. EDX analysis, however, does not have enough resolution to verify this discontinuity.

$\mathrm{X}$-ray diffraction shows that the solids of the tubes are highly disordered along with the presence of crystalline components. In the case of calcium chloride and nickel sulfate tubes, we found sodium chloride and sodium sulfate crystals, respectively, demonstrating that sodium cations can cross the osmotic membrane. In some manganese-, cobalt-, and nickel-salt experiments, we found crystals of metal oxide-hydroxide, which are known to participate in the wall structure.

\section{ASSOCIATED CONTENT}

S Supporting Information. Additional photographs, SEM images, and diffractograms are published separately. This material is available free of charge via the Internet at http://pubs.acs.org.

\section{AUTHOR INFORMATION}

\section{Corresponding Author}

*To whom correspondence should be addressed. E-mail addresses: julyan.cartwright@csic.es; bruno.escribano.salazar@ gmail.com; ignacio.sainz@iact.ugr-csic.es.

\section{Present Addresses}

${ }^{\dagger}$ Basque Center for Applied Mathematics (BCAM), Building 500

Bizkaia Technology Park, E-48160 Derio, Spain.

\section{ACKNOWLEDGMENT}

We acknowledge the support of the Spanish Ministerio de Educación y Ciencia and FEDER European Funding by means of the research projects CGL2008-06245-CO2-01 and CTQ200729861. The main instrumental analysis was performed in the Centro de Instrumentación Científica of the University of Granada and the main electron microscopy work was performed in the CEAMA centre by Isabel Sánchez, whom the authors thank. Single crystal X-ray diffraction analyses were performed by Alejandro Rodríguez-Navarro at the University of Granada; we thank him too for his help.

\section{REFERENCES}

(1) Cartwright, J. H. E.; García-Ruiz, J. M.; Novella, M. L.; Otálora, F. J. Colloid Interface Sci. 2002, 256, 351-359.

(2) Double, D. D. Phil. Trans. R. Soc. London, Ser. A 1983, 310, $53-$ 65.

(3) Fontana, M. G. Corrosion Engineering, 3rd ed.; McGraw-Hill: New York, 1986.

(4) Russell, M. J.; Hall, A. J. J. Geol. Soc. London 1997, 154, 377-402.

(5) Russell, M. J.; Kanik, I. J. Cosmol. 2010, 5, 1008-1039.

(6) Coatman, R. D.; Thomas, N. L.; Double, D. D. J.Mater. Sci. 1980, 15, 2017-2026.

(7) Collins, C.; Zhou, W.; Mackay, A.; Klinowski, J. Chem. Phys. Lett. 1998, 286, 88-92.

(8) Balköse, D.; Özkan, F.; Köktürk, U.; Ulutan, S.; Ülkü, S.; Nişli, G. J. Sol-Gel Sci. Technol. 2002, 23, 253-263.

(9) Thouvenel-Romans, S.; van Saarloos, W.; Steinbock, O. Europhys. Lett. 2004, 67, 42-48.

(10) Thouvenel-Romans, S.; Pagano, J.; Steinbock, O. Phys. Chem. Chem. Phys. 2005, 7, 2610-2615.

(11) Pagano, J.; T. Bánsági, J.; Steinbock, O. J. Phys. Chem. C 2007, $111,9324-9329$.

(12) Cartwright, J. H. E.; Escribano, B.; Khokhlov, S.; Sainz-Díaz, C. I. Phys. Chem. Chem. Phys. 2011, 13, 1030-1036.

(13) Cartwright, J. H. E.; Escribano, B.; Sainz- Díaz, C. I.; Stodieck, L. S. Langmuir 2011; DOI: 10.1021/la104193q.

(14) Martin, J. D. 2004, http://www.XPowder.com/index.html.

(15) Rodríguez-Navarro, A. J. Appl. Crystallogr. 2006, 39, 905-909.

(16) Collins, C.; Mokaya, R.; Klinowski, J. Phys. Chem. Chem. Phys. 1999, 1, 4669-4672.

(17) Makki, R.; Al-Humiari, M.; Dutta, S.; Steinbock, O. Angew. Chem. 2009, 48, 8752-8756.

(18) Hazlehurst, T. J. Chem. Educ. 1941, 18, 286-289.

(19) Tao, Y.; Zhu, B.; Chen, Z. J. Cryst. Growth 2006, 293, 382-386.

(20) Lutz, H.; Möller, H.; Schmidt, M. J. Mol. Struct. 1994, 328, 121132.

(21) Zhong, K.; Jin, P.; Chen, Q. J. Nanomater. 2006, 2006, 1-7.

(22) Jin, P.; Chen, Q.; Hao, L.; Tian, R.; Zhang, L.; Wang, L. J. Phys. Chem. B 2004, 108, 6311-6314.

(23) Lide, D. R. CRC Handbook of Chemistry and Physics, 71st ed.; CRC Press: Boston, 1991. 